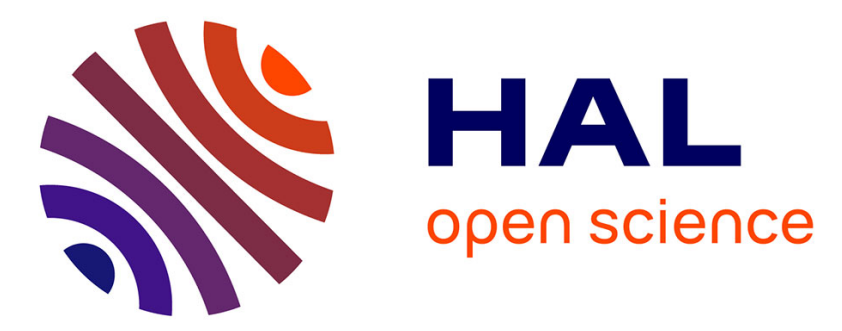

\title{
Observation of a gap in the far-infrared magneto-absorption of (TMTSF) 2ClO4: possibility of one-dimensional fluctuating superconductivity
}

\author{
H.K. Ng, T. Timusk, J.M. Delrieu, D. Jérome, K. Bechgaard, J.M. Fabre
}

\section{To cite this version:}

H.K. Ng, T. Timusk, J.M. Delrieu, D. Jérome, K. Bechgaard, et al.. Observation of a gap in the far-infrared magneto-absorption of (TMTSF) 2ClO4: possibility of one-dimensional fluctuating superconductivity. Journal de Physique Lettres, 1982, 43 (14), pp.513-519. 10.1051/jphyslet:019820043014051300 . jpa-00232084

\section{HAL Id: jpa-00232084 https://hal.science/jpa-00232084}

Submitted on 1 Jan 1982

HAL is a multi-disciplinary open access archive for the deposit and dissemination of scientific research documents, whether they are published or not. The documents may come from teaching and research institutions in France or abroad, or from public or private research centers.
L'archive ouverte pluridisciplinaire HAL, est destinée au dépôt et à la diffusion de documents scientifiques de niveau recherche, publiés ou non, émanant des établissements d'enseignement et de recherche français ou étrangers, des laboratoires publics ou privés. 
Classification

Physics Abstracts

$74.40-78.20 \mathrm{~L}$

\title{
Observation of a gap in the far-infrared magneto-absorption of (TMTSF) ${ }_{2} \mathrm{ClO}_{4}$ : possibility of one-dimensional fluctuating superconductivity $\left({ }^{*}\right)$
}

\author{
H. K. Ng $\left({ }^{1}\right)$, T. Timusk $\left({ }^{1}\right)\left({ }^{* *}\right)$, J. M. Delrieu $\left({ }^{2}\right)$, D. Jérome $\left({ }^{3}\right)\left({ }^{* * *}\right)$, K. Bechgaard $\left({ }^{4}\right)$ \\ and J. M. Fabre $\left(^{5}\right)$ \\ ( ${ }^{1}$ ) Department of Physics, McMaster University, Hamilton, Ontario L8S 4M1, Canada \\ ( ${ }^{2}$ Service de Physique des Solides, CEN Saclay, 91191 Gif sur Yvette Cedex, France \\ ( ${ }^{3}$ ) Laboratoire de Physique des Solides, Université Paris-Sud, 91405 Orsay, France \\ (4) H. C. Oersted Institute, Universitetsparken 5, DK 2100, Copenhagen, Denmark \\ $\left({ }^{5}\right)$ Laboratoire de Chimie Organique Structurale, U.S.T.L., 34060 Montpellier, France
}

(Reçu le 24 mars 1982, acceptéle 25 mai 1982)

\begin{abstract}
Résumé. - Nous présentons une méthode nouvelle de détermination de la bande interdite des supraconducteurs. Cette méthode est basée sur la variation de la transmission d'une grille du matériau à étudier dans l'infrarouge très lointain, en fonction du champ magnétique appliqué. La méthode a été testée sur des fils de plomb et de niobium et a conduit à des résultats en très bon accord avec les résultats d'effet tunnel. En ce qui concerne les cristaux organiques (TMTSF) ${ }_{2} \mathrm{ClO}_{4}$, l'application d'un champ de 0,2 tesla induit une augmentation de l'absorption optique tant que l'énergie demeure inférieure à $3,8 \mathrm{meV}$. Cette bande interdite est en bon accord avec un modèle unidimensionnel de fluctuations supraconductrices au-dessus de la température de transition de phases.

Abstract. - A new method for determining the energy gap of superconductors is presented. The far-infrared magneto-absorption is measured by looking at the change of transmission through a grid of the material upon application of a magnetic field. Excellent agreement with tunnelling data is obtained for $\mathrm{Pb}$ and $\mathrm{Nb}$ wires. On needle-like (TMTSF) ${ }_{2} \mathrm{ClO}_{4}$ crystals, the application of a field of 0.2 tesla leads to an increase of the absorption below $3.8 \mathrm{meV}$. This gap is in agreement with the proposed model of One-Dimensional fluctuating superconductivity above the phase transition temperature.
\end{abstract}

The organic charge transfer salts [1] (TMTSF) ${ }_{2} \mathrm{X}$ have attracted considerable attention in the past two years. An on-going theoretical debate is the existence of fluctuating superconductivity [2] in these quasi-one-dimensional conductors. This claim by the Orsay group [2] has being supported by tunnelling results on (TMTSF) ${ }_{2} \mathrm{PF}_{6}$ under $11 \mathrm{kbar}$ which show an anomalously large energy gap [3] (3.6 meV). An opposing view is sponsored by the UCLA-IBM group [4] who favours the

$\left({ }^{*}\right)$ A preliminary announcement of this work has been done at the APS-March meeting Dallas, Bull. Am. Phys. Soc, 27 (1982) 151.

$\left({ }^{* *}\right)$ Work supported in part by the National Science and Engineering Research Council of Canada.

${ }^{* * *}$ ) Work supported in part by the DGRST Contract 80-70165 and ATP-CNRS program 1981. 
model of a 2-dimensional conductor with very high mobility carriers in the interpretation of their results.

Although organic superconductivity was first observed [5] in (TMTSF) ${ }_{2} \mathrm{PF}_{6}$ and has thus been most extensively studied in this compound, it is (TMTSF) ${ }_{2} \mathrm{ClO}_{4}$ which seems the most interesting one since it is the only member of the charge transfer salts which becomes superconducting at ambient pressure [6], below about $1.3 \mathrm{~K}$. A detailed optical study has been done on these materials [7], but up to the present time no data exist on spectra at low frequencies in the highly conducting region i.e. at helium temperatures.

In this paper we present a new method of measuring the far-infrared (FIR) magneto-absorption in highly conducting materials applicable to very small samples. We first apply the method as a test to two well studied superconductors, lead and niobium. We also show evidence of a structure in the magneto-absorption at $3.8 \mathrm{meV}$ in (TMTSF) ${ }_{2} \mathrm{ClO}_{4}$ at $1.2 \mathrm{~K}$.

1. Principle of the method. - The methods commonly used to measure energy gaps of superconductors are transmittance and reflectance of FIR radiation [8]. In the latter large samples are needed, that cover a sizeable portion of the walls on a non resonant cavity to enhance the effect. Our method is to look at the change in transmission of a grid of regularly spaced crystals when a magnetic field is applied. This change can be related to the change in surface impedance of the wires. Transmission through a resistive wire grid has being treated theoretically by Ulrich [9], and Lewis and Casey [10] in the limit of the wire spacing $g$ being less than the wavelength. It is well known that such structures, called inductive grids, tend to be more lossy than expected from the magnitude of the surface impedance of the wires used.

Our experiments were conducted at $1.2 \mathrm{~K}$ with samples immersed in liquid helium. For $\mathrm{Pb}$ and $\mathrm{Nb}$ two arrays of parallel circular wires were laid perpendicularly to form meshes with spacing $g=200 \mu \mathrm{m}$, and wire diameters $2 a$ of 80 and $51 \mu \mathrm{m}$ respectively. The (TMTSF) ${ }_{2} \mathrm{ClO}_{4}$ crystals were glued to thin gold strips (1000/inch) on Mylar [11] with average $g$ of $\approx 180 \mu \mathrm{m}$ and $2 a$ of $\approx 80 \mu \mathrm{m}$. The size and spacing of the crystals varied and the standard deviation in $g$ and $2 a$ were $50 \mu \mathrm{m}$ and $40 \mu \mathrm{m}$ respectively. This arrangement results in an $E$-field polarized along the highly conducting $a$-axis.

The mesh was placed across a $3 \mathrm{~mm}$ diameter brass light pipe, and surrounded by a superconducting solenoid magnet. The applied magnetic field is perpendicular to the longitudinal $a$-axis of the (TMTSF) ${ }_{2} \mathrm{ClO}_{4}$ needles. The source of FIR radiation was a mercury arc lamp and measurements were made over 1 to $8 \mathrm{meV}$ range using Martin-Puplett [12] type polarizing Michelson interferometer with a doped germanium bolometer operating at $0.35 \mathrm{~K}$. Signals of $1 \mathrm{mV}$ were obtained giving $2000 / 1$ signal to noise ratios in the interferograms.

Figure 1 shows change in transmission of $\mathrm{Pb}$ and $\mathrm{Nb}$ meshes below the energy gap $2 \Delta$ with the application of a magnetic field exceeding the critical fields [13]. The uncertainty of the absolute position of the baseline is 0.01 . We estimate the uncertainty in the amplitude of the observed structure to be 0.005 . The observed change below the gap frequencies in figure 1 corresponds to the destruction of the superconducting state by the magnetic field. Below $2 \Delta$ there is no absorption in the superconducting state but above the critical field we see the normal state absorption. In our configuration this absorption is of the order of 0.02 to 0.03 . Above $2 \Delta$ the normal and superconducting states have the same surface impedance and the magneto-absorption is zero. The observed percentage change of the absorption in $\mathrm{Pb}$ is an order of magnitude larger than that calculated using the simple theory of Ulrich [9]. In this estimate we have used the surface impedance measurement of $\mathrm{Pb}$ by Brandli and Sievers [14]. For $\mathrm{Nb}$, using the theory developed in reference [9] and d.c. resistivity of $1.3 \mu \Omega \mathrm{cm}$ [15] and with Drude normal state absorption a similar order of enhancement is obtained. This magnified effect can be attributed to two physical reasons. First, geometrical surface irregularities on the $\mathrm{Pb}$ and $\mathrm{Nb}$ wires increase the absorption. Secondly, a high surface impedance can be related to impurities on the surface such as nitrogen 


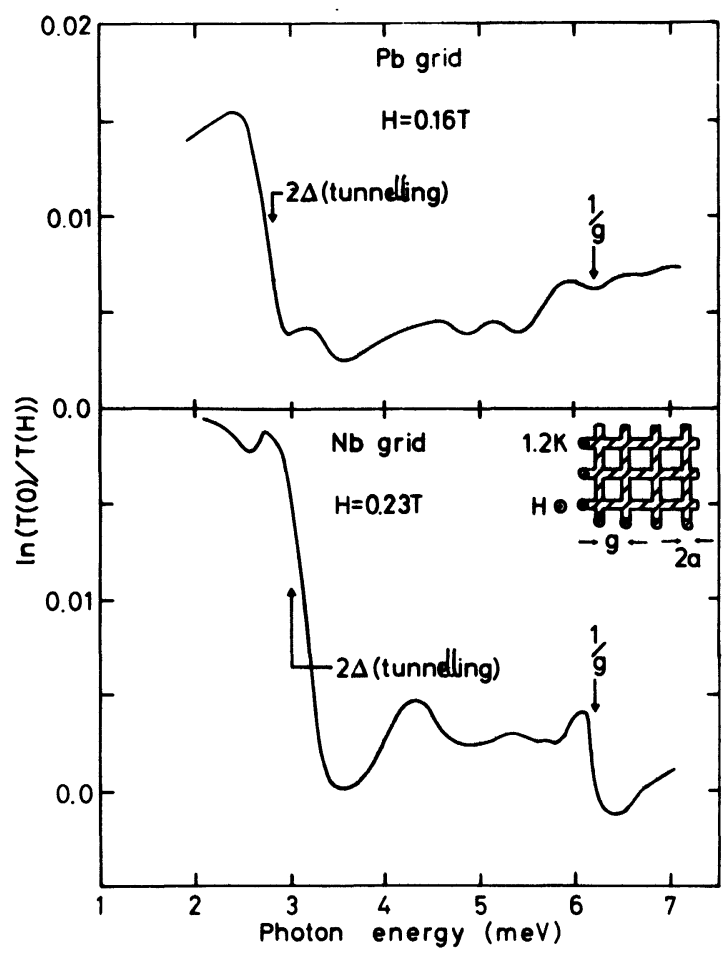

Fig. 1. - Magneto-absorption of $\mathrm{Nb}$ and $\mathrm{Pb}$ grids. The $\log _{\mathrm{e}}$ of the ratio of the transmission in the superconducting state to the normal state is plotted as a function of frequency. In both cases there is an onset of magneto-absorption below the energy gap as the superconductivity is destroyed by the magnetic field. The arrow marked $1 / g$ denotes the position of grid spacing frequency.

and oxygen in the case of $\mathrm{Nb}$. Runs on etched wires give much weaker signals in accord with these ideas.

2. Experimental data on (TMTSF) ${ }_{2} \mathrm{ClO}_{4^{\circ}}$ - The technique outlined above is applied to (TMTSF $)_{2} \mathrm{ClO}_{4}$ crystals. In figure 2 we show the magneto-absorption signal in a $0.2 \mathrm{~T}$ field. The outstanding feature in this curve is an increase in the absorption of $0.03 \pm 0.005$ below $3.8 \pm 0.5 \mathrm{meV}$ observed when a field of $0.2 \mathrm{~T}$ is applied at $1.2 \mathrm{~K}$. This feature was also observed in a lower field $(0.1 \mathrm{~T})$ and found to have a similar position and strength. We have used samples with different grid spacings $(g \sim 600 \mu \mathrm{m}$ and $2 a=150$ to $250 \mu \mathrm{m})$ and different sources : Copenhagen and Montpellier. In each case we observe the structure shown in figure 2 . The structure shown in figures 1 and 2 were reproducible and observed in all samples tested. As an additional check we placed a pair of $\mathrm{Nb}$ grids in series tilted to avoid Fabry-Perot fringes and found the effect doubled as expected.

The structure shown in figures 1 and 2 for the two conventional superconductors and the (TMTSF) ${ }_{2} \mathrm{ClO}_{4}$ are similar in several ways. The sign of the magneto-absorption is the same in all three spectra, the amplitude is of the same order of magnitude (0.02-0.03) and the energy of the magneto-absorption feature falls in the same range $(3$ to $4 \mathrm{meV})$. The magnetic field of $\sim 0.2 \mathrm{~T}$ necessary to produce an observable magneto-absorption is also the same. However there are two important differences. First for $\mathrm{Nb}$ and $\mathrm{Pb}$ the ratio between the gap observed in FIR and $k_{\mathrm{B}} T_{\mathrm{c}}$ is of the order of the BCS ratio, i.e. 3.7 [13], whereas for (TMTSF) ${ }_{2} \mathrm{ClO}_{4}$ which is known to show superconductivity around $1.3 \mathrm{~K}[16] 2 \Delta / k T_{\mathrm{c}}$ amounts to about 30 . Moreover the $3.8 \mathrm{meV}$ 


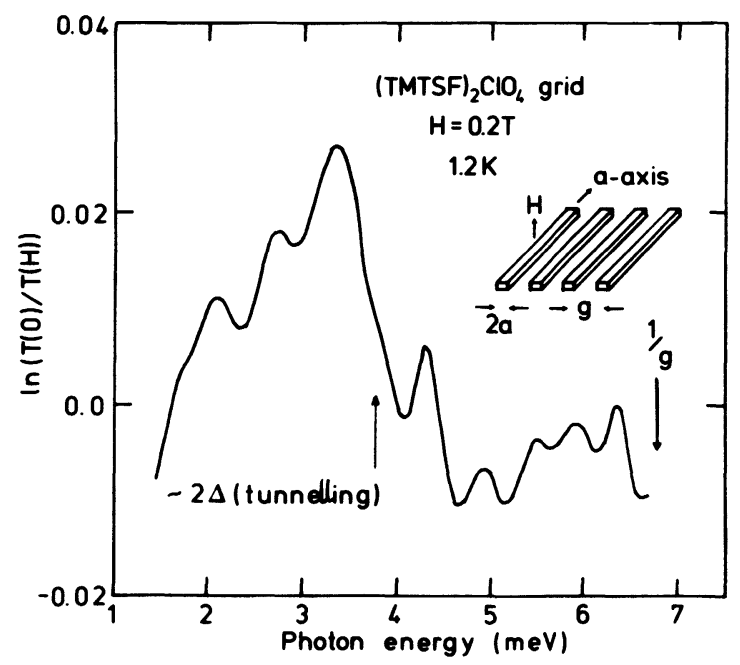

Fig. 2. - Magneto-absorption of a grid of (TMTSF $)_{2} \mathrm{ClO}_{4}$. The electromagnetic radiation is polarized along the long $a$-axis of the crystals, the static magnetic field is normal to this axis. There is a pronounced onset of magneto-absorption below $3.8 \mathrm{meV}$ that we associate with an energy gap in the electronic density of states.

FIR anomaly can be observed at. a temperature so close to the transition temperature that in the case of a conventional superconductor the gap should exhibit a very small amplitude.

Furthermore, following the measurement of the gap below $T_{c}$ derived from recent specific heat experiments [17], the ratio $2 \Delta / k T_{\mathrm{c}}$ equals about 4 , a value which is more in the line of conventional strong coupling superconductors. Therefore, a conventional three-dimensional model does not allow us to ascribe the $3.8 \mathrm{meV}$ FIR anomaly to the observed superconducting properties observed below $1.3 \mathrm{~K}$.

3. Discussion. - One possibility is that the FIR feature has no connection with the superconducting properties of this material and results from other phenomena. We can rule out phonons and librational modes by the strong magnetic field dependence of the effect. We can also eliminate ordinary magnetoresistance. Using Chaikin's experimental value for the d.c. magnetoresistance [18] and Drude theory to derive the corresponding IR conductivity under magnetic field, we estimate a contribution to magneto-absorption of less than a tenth of our observed signal. Moreover this effect is likely to be even much smaller since the magnetoresistance should be small when the irradiation frequency is larger than the cyclotron frequency, as encountered here.

The existence of a spin density wave (SDW) state has been proposed by Walsh et al. [19] at a relatively low field and at a temperature below $4 \mathrm{~K}$ or so. Under these conditions they observe a resonance similar to an antiferromagnetic one. This antiferromagnetic resonance has parameters in the same range as (TMTSF) ${ }_{2} \mathrm{PF}_{6}[20]$ and may in analogy possess a transition temperature in the neighbourhood of $12 \mathrm{~K}$. Using the BCS model, we expect a SDW gap in the range of $4 \mathrm{meV}$ for (TMTSF) ${ }_{2} \mathrm{PF}_{6}$ and presumably a slightly smaller value of the gap for (TMTSF) ${ }_{2} \mathrm{ClO}_{4}$ following the data of Walsh et al. [19].

As a first attempt we assume $T_{\mathrm{SDW}} \approx 6 \mathrm{~K}$ which corresponds to the temperature of the resistance minimum observed in some (TMTSF) ${ }_{2} \mathrm{ClO}_{4}$ samples [21] and we use the field dependence of $T_{\text {SDw }}$ as measured in (TMTSF) ${ }_{2} \mathrm{PF}_{6}$ [22], namely $\frac{1}{T_{\mathrm{c}}} \frac{\mathrm{d} T_{\mathrm{c}}}{\mathrm{d} H}<0.1 \mathrm{~T}^{-1}$. Therefore, a field of 
$0.2 \mathrm{~T}$ should increase a SDW gap by at most $2 \%$. The two following situations must be considered :

(i) If the gap edge is sharp, such a small shift should lead to a sharp peaking of the magnetoabsorption at the gap edge itself with a width only $2 \%$ that of the $3.8 \mathrm{meV}$ gap. In contrast, figure 2 shows a broad magneto-absorption about $2 \mathrm{meV}$ wide.

(ii) If the gap edge is smeared (by finite temperature effects, distribution of the gap amplitude, etc...) the relative change of the absorption under magnetic field is expected to be of the order of magnitude of the relative increase of the gap, namely $2 \%$.

However the comparison between the data of (TMTSF) ${ }_{2} \mathrm{ClO}_{4}$, figure 2 and the data of regular superconductors, figure 1 , shows that the change of absorption of (TMTSF) ${ }_{2} \mathrm{ClO}_{4}$ is roughly what is expected if the sample undergoes a transition from the superconducting to the normal state; this is admittedly much larger than the $2 \%$ increase which may be accounted for by a gap originating from SDW.

As a second attempt to explain the FIR anomaly by a SDW gap we may use a different set of experimental results. In (TMTSF) ${ }_{2} \mathrm{PF}_{6}$ where a SDW has been observed directly by susceptibility [22], the NMR line of ${ }^{77} \mathrm{Se}$ broadens up to a point which makes this line vanish [23] ( $\gtrsim 20$ times the non-magnetic state linewidth). In (TMTSF) ${ }_{2} \mathrm{ClO}_{4}$ the ${ }^{77} \mathrm{Se}$ resonance line exhibits a relatively small broadening [24] (34 Oe in the normal state at $20 \mathrm{~K}$ and $54 \mathrm{Oe}$ just before the SDW transition at $\approx 4 \mathrm{~K}$ in a field of $5 \mathrm{~T}$ ). Consequently, the high field SDW transition observed in (TMTSF) $)_{2} \mathrm{ClO}_{4}$ is expected to occur around $1 \mathrm{~K}$ under $0.2 \mathrm{~T}$, together with a small amplitude of the gap, about $1 / 12$ that observed in (TMTSF) ${ }_{2} \mathrm{PF}_{6}$, i.e. $\approx 0.3 \mathrm{meV}$. Hence, the SDW temperature may possibly increase under magnetic field at a rate of $\frac{1}{T_{\mathrm{c}}} \frac{\mathrm{d} T_{\mathrm{c}}}{\mathrm{d} H} \approx 1 \mathrm{~T}^{-1}$, starting from about $1 \mathrm{~K}$ in zero field. In that case a magneto-absorption somewhat larger than the value inferred from the (TMTSF) ${ }_{2} \mathrm{PF}_{6}$ data is expected, but the FIR anomaly should be visible around $0.3 \mathrm{meV}$, in contradiction with the data in figure 2.

This analysis shows that the possible existence of a SDW at low field in (TMTSF) ${ }_{2} \mathrm{ClO}_{4}$ cannot explain the gap of $3.8 \mathrm{meV}$ displayed in figure 2 . Since the ${ }^{77} \mathrm{Se}$ resonance line is a very sensitive probe of the amplitude of the SDW magnetization it is difficult to reconcile the small ${ }^{77} \mathrm{Se}$ line broadening with the large magnetic order parameter determined recently by antiferromagnetic resonance in (TMTSF) ${ }_{2} \mathrm{ClO}_{4}[19]$. The hypothetically small SDW gap suggested by the ${ }^{77} \mathrm{Se}$ resonance and the corresponding low phase transition temperature are in contradiction with the amplitude of the zero field antiferromagnetic resonance frequency and its apparent field independence [19].

There is another interesting possible model to resolve this contradiction : the presence of one-dimensional fluctuating superconductivity with pairing in the triplet state; in analogy to helium-3 [25], the anisotropy leads to zero field resonance frequencies which can be taken for a SDW resonance. Since there is no spatial modulation of magnetization in the superconducting triplet state there would be no broadening of the ${ }^{77}$ Se NMR line, except for a weak change due to the hyperfine coupling of the electronic spin to the nuclear spin which is slightly increased in the triplet superconducting state [26].

Finally we feel justified to claim that the FIR anomaly observed with a (TMTSF) ${ }_{2} \mathrm{ClO}_{4}$ grid at $3.8 \mathrm{meV}$ is related neither to the superconducting state establishing at very low temperature $(T<1.3 \mathrm{~K})$ in this compound nor to a SDW state. Consequently we attribute the FIR anomaly to the development of short range one-dimensional superconducting pairing [2], with a characteristic pairing energy $2 \Delta=3.8 \mathrm{meV}(42 \mathrm{~K})$. 1-D short range order can induce a marked depression of the density of states $N\left(E_{\mathrm{F}}\right)$ at the Fermi surface in a temperature domain extending from $T_{3}$ up to a temperature $T_{1}$ which must be " in speaking terms " with the size of the 1-D pairing energy $\left(2 \Delta \approx k T_{1}\right)$. This is the so-called " pseudo-gap effect ». There are several other experimen- 
tal evidences for the existence of a large pseudo-gap in the family of quasi 1-D superconductors $(\text { TMTSF })_{2} \mathrm{X}$.

(i) The direct observation of a marked gap of width $\approx 3.6 \mathrm{meV}$ in the Fermi level density of states of (TMTSF) ${ }_{2} \mathrm{PF}_{6}$ under a pressure of $12 \mathrm{kbar}$ via electron tunnelling experiments [3].

(ii) The observation of an important drop of the thermal conductivity below $40 \mathrm{~K}$ in (TMTSF) ${ }_{2} \mathrm{PF}_{6}$ under $12 \mathrm{kbar}$ [27] and in (TMTSF) ${ }_{2} \mathrm{ClO}_{4}$ at ambient pressure [28], which is removed by the application of a magnetic field.

(iii) The magnetic field dependence of $N\left(E_{\mathrm{F}}\right)$ in (TMTSF) ${ }_{2} \mathrm{ClO}_{4}$ (at high fields $H \gtrsim 10 \mathrm{kOe}$ ) as derived from the electronic contribution to the low temperature specific heat [17].

(iv) The recent observation of an anomaly in the electron tunnelling data of (TMTSF) ${ }_{2} \mathrm{ClO}_{4}$ at low temperature which is consistent with the far-infrared data of the present work [29].

Moreover, the pseudo-gap at low temperature is not restricted to the pressure range in which a 3-D ordered superconductivity is stable, since far-infrared reflectance experiments performed on (TMTSF) ${ }_{2} \mathrm{PF}_{6}$ under ambient pressure at $T \approx 25 \mathrm{~K}$ have also clearly established the existence of a gap in the $30-50 \mathrm{~cm}^{-1}$ domain [7] (while a SDW ground state becomes stable below $12 \mathrm{~K}$ ). Besides the accumulating experimental observations in favour of short range 1-D superconducting ordering at high temperature in the organic superconductors (TMTSF) ${ }_{2} \mathrm{X}$, this work presents additional arguments based on spectroscopic data. Studies of the FIR spectra in terms of the temperature and magnetic fields are now in progress.

\section{References}

[1] Bechgaard, K., Jacobsen, C. S., Mortensen, K., Pedersen, H. J. and Thorup, N., Solid State Commun. 33 (1980) 1119.

[2] JÉRome, D., Proceedings of the International Conference on Low Dimensional Conductors, Boulder 1981, Mol. Cryst. Liq. Cryst. 79 (1982) 155.

[3] More, C., Roger, G., Sorbier, J. P., Jérome, D., Ribault, M. and BechgaArd, K., J. Physique-Lett. 42 (1981) L-313.

[4] Greene, R. L., Haen, P., Huang, S. Z., Engler, E. M., Choi, M. Y. and Chaikin, P. M., Proceedings of the International Conference on Low Dimensional Conductors, Boulder 1981, Mol. Cryst. Liq. Cryst. 79 (1982) 183.

[5] Jérome, D., Mazaud, A., Ribault, M. and Bechgaard, K., J. Physique-Lett. 41 (1980) L-95.

[6] Bechgaard, K., Carneiro, K., Olsen, M., Rasmussen, F. B. and Jacobsen, C. S., Phys. Rev. Lett. 46 (1981) 832.

[7] JACobsen, C. S., TANner, D. B. and BechgaARD, K., Proceedings of the International Conference on Low Dimensional Conductors, Boulder 1981, Mol. Cryst. Liq. Cryst. 79 (1982) 25.

[8] Ginsberg, D. M., Richards, P. L. and Tinkham, M., Phys. Rev. Lett. 3 (1957) 337. See also

Palmer, L. H. and Tinkham, M., Phys. Rev. 165 (1968) 588 ;

McKnight, S. W., Bean, B. L. and Perkowitz, S., Phys. Rev. B 19 (1979) 1437.

[9] UlRiCH, R., Infrared Phys. 7 (1967) 37, see also

Moeller, K. D. and Rotschild, W. G., Far-Infrared Spectroscopy (Wiley) 1971, p. 93.

[10] Lewis, E. A. and CASey, J. P., J. Appl. Phys. 23 (1952) 605.

[11] Mylar is a trade name for polyethylene terephthalate.

[12] Martin, P. H. and Puplett, E., Infrared Phys. 10 (1969) 105.

[13] Mersevey, R. and Schwartz, B. B., Superconductivity, R. D. Parks, ed. (Dekker, New York) 1969.

[14] Brandli, G. and Sievers, A. J., Phys. Rev. B 5 (1972) 3550.

[15] Our measurement using 4-probe technique.

[16] BechgaARd, K., Mol. Cryst. Liq. Cryst. 79 (1982) 1. 
[17] Garoche, P., Brusetti, R., Jérome, D. and Bechgand, K., J. Physique-Lett. 43 (1982) L-147.

[18] Chaikin, P. M., Mu-Yong Choi, Haen, P., Engler, E. M. and Greene, R. L., Mol. Cryst. Liq. Cryst. 79 (1982) 79.

[19] Walsh, W. M., Bull. Am. Phys. Soc. 27 (1982) 150.

[20] Torrance, J. B., Pedersen, H. J. and Bechgaard, K., ibid.

[21] Parkin, S. S. P., Ribault, M., Jérome, D. and Bechgaard, K., J. Phys. C 14 (1981) 5305.

[22] Mortensen, K., Tomkiewicz, Y., Schultz, T. D. and Engler, E. M., Phys. Rev. Lett. 46 (1981) 1234.

[23] Andrieux, A., JÉrome, D., Bechgaard, K., J. Physique-Lett. 42 (1981) L-87.

[24] Takahashi, T., Jérome, D., BechgaARd, K., J. Physique-Lett., to be published (1982).

[25] Legget, A. J., Rev. Mod. Phys. 47 (1975) 331.

[26] Shing Takagi, Prog. Theor. Phys. 51 (1974) 1998.

[27] DJuReK, D., Jérome, D., Weyl, C. and BechgaARd, K., to be published (1982).

[28] Djurek, D., Prester, M., Jérome, D. and BechgaArd, K., J. Phys. C. Lett., in press.

[29] Sorbier, J. P., private communication. 